\title{
A G-KKM TYPE THEOREM AND ITS APPLICATIONS TO MINIMAX INEQUALITIES ON G-CONVEX SPACES
}

\author{
MOHAMMAD S.R. CHOWDHURY \\ Dalhousie University \\ Department of Mathematics, Statistics and Computing Science \\ Halifax, Nova Scotia, Canada B3H 3 J5
}

(Received September, 1996; Revised January, 1998)

A G-KKM type theorem is obtained on G-convex spaces. As application, a generalization of Ky Fan's minimax inequality to non-compact sets on G-convex spaces is first obtained. As special cases of this minimax inequality, some new minimax inequalites are obtained. Four fixed point theorems and four equivalent formulations of the second minimax inequality are also obtained.

Key words: G-Convex Spaces, G-Convex Sets, G-Convex Hulls, GKKM Theorem, Minimax Inequality, Lower Semi-Continuous and Upper Semi-Continuous Maps.

AMS subject classifications: $47 \mathrm{H} 04,47 \mathrm{H} 05,47 \mathrm{H} 09,47 \mathrm{H} 10,54 \mathrm{C} 60$.

\section{Introduction and Preliminaries}

If $X$ is a non-empty set, we denote by $2^{X}$ the family of all subsets of $X$, by $\langle X\rangle$ or by $\mathcal{F}(X)$ the family of all non-empty finite subsets of $X$, and by $|X|$ the cardinality of $X$. If $X$ is a subset of a vector space, $c o(X)$ denotes the convex hull of $X . \Delta_{n}$ denotes the standard $n$-simplex $\operatorname{co}\left\{e_{0}, e_{1}, \ldots, e_{n}\right\}$, where $e_{i}$ is the $(i+1)$ th unit vector in $\mathbb{R}^{n+1}$. If $X$ is a topological space, then $c l_{X}(A)$ denotes the closure of $A$ in $X$ and int $_{X}(A)$ denotes the interior of $A$ in $X$.

Let $X$ be a topological space and $D$ be a non-empty subset of $X$. Suppose that $\Gamma:\langle D\rangle \rightarrow 2^{X} \backslash\{\emptyset\}$ is a mapping such that $(a)$ for each $A, B \in\langle D\rangle, A \subset B$ implies $\Gamma(A) \subset \Gamma(B)$ and $(b)$ for each $A \in\langle D\rangle$, say $A=\left\{a_{0}, \ldots, a_{1}, \ldots, a_{n}\right\}$ there exists a continuous function $\phi: \Delta_{n} \rightarrow \Gamma(A)$ such that for each $0 \leq i_{0}<\ldots<i_{k} \leq n, \phi\left(\operatorname{co}\left\{e_{i_{0}}, \ldots\right.\right.$ $\left.\left.e_{i_{k}}\right\}\right) \subset \Gamma\left(\left\{a_{i_{0}}, \ldots, a_{i_{k}}\right\}\right)$. Then $(X, D ; \Gamma)$ is called a $G$-convex space [12]. When $D=$ $X$, we shall write $(X ; \Gamma)$ in place of $(X, X ; \Gamma)$.

Let $(X, D ; \Gamma)$ be a $G$-convex space and $K \subset X$. Then $(i) K$ is $G$-convex [12] if for each $A \in\langle D\rangle, A \subset K$ implies $\Gamma(A) \subset K$; (ii) the G-convex hull [12] of $K$, denoted by 
$G$-co $(K)$, is the set $\cap\{B \subset X: B$ is a $G$-convex subset of $X$ containing $K\}$.

As noted in [12], the concept of a $G$-convex space is a generalization of convexity in vector spaces, Horvath's pseudo-convex spaces [8] and $c$-structure [9], $H$-spaces [13] and many others.

The following definition is a slight modification of Park's definition in [11].

Let $X$ and $Y$ be subsets of a $G$-convex space $(E, \Gamma)$ such that $G$-co $(X) \subset Y$, and suppose for each $x \in E,\{x\}$ is $G$-convex, i.e., $\Gamma(\{x\})=\{x\}$. Suppose that for each $A \in\langle E\rangle, G-\operatorname{co}(A)$ is compact and $\Gamma(A)=G-\operatorname{co}(A)$. Then $F: X \rightarrow 2^{Y}$ is called a $G$ $K K M$-map if for each $A \in\langle X\rangle, G-\operatorname{co}(A) \subset \cup_{x \in A} F(x)$.

Note that if $F$ is a G-KKM-map, then $x \in F(x)$ for all $x \in X$, since $G$-co $(\{x\})=$ $\Gamma(\{x\})=\{x\} \subset F(x)$.

Theorem 1.1: Let $(E, \Gamma)$ be a $G$-convex space such that, for each $x \in E,\{x\}$ is $G$ convex (i.e., $\Gamma\{x\}=\{x\}$ ), and for each $A \in\langle E\rangle, G-c o(A)$ is compact and $\Gamma(A)=G$ $c o(A)$. Let $X$ and $Y$ be non-empty subsets of $E$ such that $X \subset Y$ and $Y$ is $G$-convex. Suppose $F: X \rightarrow 2^{Y}$ is such that

(a) $F$ is a G-KKM-map;

(b) for each $x \in X, F(x)$ is closed in $Y$;

(c) there exists $x_{0} \in X$ such that $F\left(x_{0}\right)$ is compact.

Then $\cap_{x \in X} F(x) \neq \emptyset$.

Proof: We shall first show that the family $\{F(x): x \in X\}$ has the finite intersection property. For any $n \in \mathbb{N}$, consider any finite subset $A=\left\{y_{0}, y_{1}, \ldots, y_{n}\right\}$ of $X$. Since $(E, \Gamma)$ is a $G$-convex space, there is a continuous function $\psi: \Delta_{n} \rightarrow \Gamma(A)$ such that for each $0 \leq i_{0}<\ldots<i_{k} \leq n, \psi\left(\operatorname{co}\left\{e_{i_{0}}, \ldots, e_{i_{k}}\right\}\right) \subset \Gamma\left(\left\{y_{i_{0}}, \ldots, y_{i_{k}}\right\}\right)$. Since $F$ is a G-KKM$\operatorname{map}, \Gamma(A)=G-\operatorname{co}\left(\left\{y_{0}, y_{1}, \ldots, y_{n}\right\}\right) \subset \cup_{i=0}^{n} F\left(y_{i}\right)$.

Let $G_{i}=\psi^{-1}\left(F\left(y_{i}\right)\right)$ for all $i=0,1, \ldots, n$. Then each $G_{i}$ is closed in $\Delta_{n}$ as $F\left(y_{i}\right)$ is closed and $\psi$ is continuous.

Now, for each $0 \leq i_{0}<\ldots<i_{k} \leq n, \operatorname{co}\left(\left\{e_{i_{0}}, \ldots, e_{i_{k}}\right\}\right) \subset \psi^{-1}\left(\Gamma\left(\left\{y_{i_{0}}, \ldots, y_{i_{k}}\right\}\right)\right)=$ $\psi^{-1}\left(G-\operatorname{co}\left(\left\{y_{i_{0}}, \ldots, y_{i_{k}}\right\}\right)\right) \subset \psi^{-1}\left(\cup_{j=0}^{k} F\left(y_{i}\right)\right)=\cup_{j=0}^{k} \psi^{-1}\left(F\left(y_{i_{j}}\right)\right)=\cup_{j=0}^{k} G_{i_{j}}$. Therefore, by the KKM theorem [10], $\cap_{i=0}^{n} G_{i} \neq \emptyset$.

Let $x^{\prime} \in \cap_{i=0}^{n} G_{i}$. Then $x^{\prime} \in G_{i}$ for all $i=0,1, \ldots, n$. But $G_{i}=\psi^{-1}\left(F\left(y_{i}\right)\right)$, so that $\psi\left(x^{\prime}\right) \in F\left(y_{i}\right)$ for all $i=0,1, \ldots, n$. Hence $\psi\left(x^{\prime}\right) \in \cap_{i=0}^{n} F\left(y_{i}\right)$. Thus $\{F(x)$ : $x \in X\}$ has the finite intersection property. Therefore, $\left\{F\left(x_{0}\right) \cap F(x): x \in X\right\}$ has the finite intersection property. Consequently, $\cap_{x \in X}\left(F\left(x_{0}\right) \cap F(x)\right) \neq \emptyset$. Hence $\cap_{x \in X} F(x) \neq \emptyset$.

The following result is Lemma 2.1 of Tan and Zhang in [16].

Lemma A: Let $(E, \Gamma)$ be a G-convex space and $X$ be a non-empty subset of $E$. Then

(1) $G-\operatorname{co}(X)$ is a $G$-convex subset of $E$;

(2) $G$-co $(X)$ is the smallest $G$-convex subset of $E$ containing $X$;

(3) $G-c o(X)=\cup\{G-c o(A): A \in\langle X\rangle\}$.

We now state the following generalized version of the celebrated $1972 \mathrm{Ky} \mathrm{Fan's}$ minimax inequality [7, Theorem 1] due to Chowdhury and Tan [5, Theorem 1].

Theorem A: Let $E$ be a topological vector space, $X$ be a non-empty convex subset of $E, h: X \rightarrow \mathbb{R}$ be lower semicontinuous on co $(A)$ for each $A \in \mathcal{F}(X)$. Let $f: X \times$ $X \rightarrow \mathbb{R} \cup\{-\infty,+\infty\}$ be such that

(a) for each $A \in \mathcal{F}(X)$ and each fixed $x \in c o(A), y \mapsto f(x, y)$ is lower semicontinuous on $\operatorname{co}(A)$; 
(b) for each $A \in \mathscr{F}(X)$ and each $y \in c o(A), \min _{x \in A}[f(x, y)+h(y)-h(x)] \leq 0$;

(c) for each $A \in \mathscr{F}(X)$ and each $x, y \in \operatorname{co}(A)$ and every net $\left\{y_{\alpha}\right\}_{\alpha \in \Gamma}$ in $X$ converging to $y$ with

$$
f\left(t x+(1-t) y, y_{\alpha}\right)+h\left(y_{\alpha}\right)-h(t x+(1-t) y) \leq 0 \text { for all } \alpha \in \Gamma \text { and all } t \in[0,1] \text {, }
$$

we have $f(x, y)+h(y)-h(x) \leq 0$;

(d) there exist a non-empty closed and compact subset $K$ of $X$ and $x_{0} \in K$ such that $f\left(x_{0}, y\right)+h(y)-h\left(x_{0}\right)>0$ for all $y \in X \backslash K$.

Then there exists $\widehat{y} \in K$ such that $f(x, \widehat{y}) \leq h(x)-h(\widehat{y})$ for all $x \in X$.

The purpose of this paper is to present a further generalization of Theorem A into $G$-convex spaces. In Section 2 of this paper, we shall obtain a G-KKM type theorem which is a generalization of Lemma 2 in [5] into G-convex spaces. Thus our G-KKM type theorem will also extend and improve the classical KKM theorem [10] into Gconvex spaces. In Section 3, we shall apply the G-KKM type theorem to generalize Theorem A [5, Theorem 1] and other minimax inequalities in [5] and, in turn, to generalize Ky Fan's minimax inequality [7, Theorem 1] into $G$-convex spaces. In Section 4 , we shall obtain four equivalent formulations of the second minimax inequality in Section 3. Four fixed point theorems will also be formulated, each of which either will be equivalent to this second minimax inequality or will follow from it.

\section{A G-KKM Type Theorem on G-Convex Spaces}

In this section we shall prove the following result of G-KKM type, which generalizes Lemma 2 in [5] into $G$-convex spaces.

Theorem 2.1: Let $(E, \Gamma)$ be a $G$-convex space such that, for each $x \in E,\{x\}$ is $G$ convex (i.e., $\Gamma(\{x\})=\{x\}$ ), and for each $A \in\langle E\rangle, G-c o(A)$ is compact and $\Gamma(A)=$ $G$-co $(A)$. Let $X$ be a non-empty $G$-convex subset of $E$. Let $F: X \rightarrow 2^{X}$ be a $G$-KKMmap such that

(a) $c_{X} F\left(x_{0}\right)$ is compact for some $x_{0} \in X$;

(b) for each $A \in\langle X\rangle$ with $x_{0} \in A$ and for each $x \in G-c o(A), F(x) \cap G-c o(A)$ is closed in $G-c o(A)$; and

(c) for each $A \in\langle X\rangle$ with $x_{0} \in A$,

$$
\left(c l_{X}\left(\bigcap_{x \in G-\operatorname{co}(A)} F(X)\right)\right) \cap G-\operatorname{co}(A)=\left(\bigcap_{x \in G-c o(A)} F(x)\right) \cap G-\operatorname{co}(A) .
$$

Then $\bigcap_{x \in X} F(x) \neq \emptyset$.

Proof: Fix an $A \in\langle X\rangle$ with $x_{0} \in A$. Define $G_{A}: G-\operatorname{co}(A) \rightarrow 2^{G-\operatorname{co}(A)}$ by $G_{A}(x)=$ $F(x) \cap G-c o(A)$ for each $x \in G-c o(A)$.

Now, for each $x \in G$-co $(A), G_{A}(x)$ is non-empty, since $F$ is a G-KKM map, and closed in $G-c o(A)$ by $(b)$. Note that $G-c o(A)=\Gamma(A)$ is compact. Thus each $G_{A}(x)$ is also compact. For each $B \in\langle G-c o(A)\rangle$ we have $B \in\langle X\rangle$ as $G-c o(A) \subset X$, and so $G-\operatorname{co}(B) \subset \cup_{x \in B} F(x)$. But $G-\operatorname{co}(B) \subset G-\operatorname{co}(A)$. It follows that

$$
G-\operatorname{co}(B) \subset\left(\bigcup_{x \in B} F(x)\right) \cap G-\operatorname{co}(A)=\bigcup_{x \in B}(F(x) \cap G-c o(A))=\bigcup_{x \in B} G_{A}(x) .
$$

Thus $G_{A}$ is a G-KKM-map on $G-c o(A)$. Hence, by Theorem 1.1, we have

$$
\bigcap_{x \in G-\operatorname{co}(A)} G_{A}(x) \neq \emptyset \text {, i.e., } \bigcap_{x \in G-c o(A)} F(x) \cap G-c o(A) \neq \emptyset \text {. }
$$


Let $\left\{E_{i}\right\}_{i \in I}$ be the family of all $G$-convex hulls of finite subsets of $X$ containing the point $x_{0}$, partially ordered by $\subset$.

Now, for each $i \in I$, let $E_{i}=G-\operatorname{co}\left(A_{i}\right)$, where $A_{i} \in\langle X\rangle_{0}$ and $\langle X\rangle_{0}$ is the family of all non-empty finite subsets of $X$ containing the point $x_{0}$.

By (2.1), for each $i \in I, \bigcap_{x \in E_{i}} F(x) \cap E_{i} \neq \emptyset$. For each $i \in I$, fix any $u_{i} \in$ $\bigcap_{x \in E_{i}} F(x) \cap E_{i}$ and let

$$
\Phi_{i}=\left\{u_{j} \mid j \geq i, j \in I\right\} .
$$

Clearly, $(i)\left\{\Phi_{i} \mid i \in I\right\}$ has the finite intersection property and (ii) $\Phi_{i} \subset F\left(x_{0}\right)$ for all $i \in I$. Then $c l_{X} \Phi_{i} \subset c l_{X} F\left(x_{0}\right)$ for all $i \in I$. By compactness of $c_{X} F\left(x_{0}\right)$, $\bigcap_{i \in I^{c l}} \Phi_{i} \neq \emptyset$. Choose any $\widehat{x} \in \bigcap_{i \in I^{c l}}{ }_{X} \Phi_{i}$. Note that for any $i \in I$ and all $j \in I$ with $j \geq i$,

Therefore,

$$
u_{j} \in \bigcap_{x \in E_{j}} F(x) \cap E_{j}\left(\subset \bigcap_{x \in E_{i}} F(x) \cap E_{j}\right) \subset \bigcap_{x \in E_{i}} F(x) .
$$

$$
\Phi_{i} \subset \bigcap_{x \in E_{i}} F(x) .
$$

Now, for any $x \in X$, there exists $i_{0} \in I$ such that $x, \widehat{x} \in E_{i_{0}}$. Therefore, for all $i \geq$ $i_{0}$, we have $x, \widehat{x} \in E_{i_{0}} \subset E_{i}$ and

$$
\begin{gathered}
\widehat{x} \in E_{i} \cap c l_{X} \Phi_{i} \subset E_{i} \cap\left(\operatorname{cl}_{X} \bigcap_{z \in E_{i}} F(z)\right) \\
=\left(\bigcap_{z \in E_{i}} F(z)\right) \cap E_{i} \quad(\text { by }(c)) \\
=\left(\bigcap_{z \in E_{i}} F(z) \cap E_{i}\right) \subset F(x) .
\end{gathered}
$$

Thus, $\widehat{x} \in F(x)$ for all $x \in X$. Hence $\bigcap_{x \in x} F(x) \neq \emptyset$.

\section{Applications of the G-KKM Type Theorem to Minimax Inequalities in G-Convex Spaces}

In this section we shall obtain several minimax inequalities as applications of the GKKM type theorem.

We shall first establish the following minimax inequality.

Theorem 3.1: $\quad$ Let $(E, \Lambda)$ be a G-convex space such that, for each $x \in E,\{x\}$ is G-convex (i.e., $\Gamma(\{x\})=\{x\})$, and for each $A \in\langle E\rangle, G$-co $(A)$ is compact and $\Gamma(A)=G-\operatorname{co}(A)$. Let $X$ be a non-empty $G$-convex subset of $E$ and $h: X \rightarrow \mathbb{R}$ be lower semicontinuous on $G-\operatorname{co}(A)$ for each $A \in\langle X\rangle$. Let $f: X \times X \rightarrow \mathbb{R} \cup\{-\infty,+\infty\}$ be such that

(a) for each $A \in\langle X\rangle$ and each fixed $x \in G-c o(A), y \mapsto f(x, y)$ is lower semicontinuous on $G$-co $(A)$;

(b) for each $A \in\langle X\rangle$ and each $y \in G-c o(A), \min _{x \in A}[f(x, y)+h(y)-h(x)] \leq 0$;

(c) for each $A \in\langle X\rangle$ and each $x, y \in G-\operatorname{co}(A)$ and every net $\left\{y_{\alpha}\right\}_{\alpha \in \Gamma}$ in $X$ converging to $y$ with

$$
f\left(z, y_{\alpha}\right)+h\left(y_{\alpha}\right)-h(z) \leq 0 \text { for all } \alpha \in \Gamma \text { and all } z \in G-c o(\{x, y\}),
$$


we have $f(x, y)+h(y)-h(x) \leq 0$;

(d) there exist a non-empty closed and compact subset $K$ of $X$ and $x_{0} \in K$ such that $f\left(x_{0}, y\right)+h(y)-h\left(x_{0}\right)>0$ for all $y \in X \backslash K$.

Then there exists $\widehat{y} \in K$ such that $f(x, \widehat{y}) \leq h(x)-h(\widehat{y})$ for all $x \in X$.

Proof: Define $F: X \rightarrow 2^{X}$ by

$$
F(x)=\{y \in X: f(x, y)+h(y)-h(x) \leq 0\} \text { for each } x \in X .
$$

If $F$ is not a G-KKM map, then for some finite subset $A=\left\{x_{1}, \ldots, x_{n}\right\}$ of $X$, there exists $\bar{y} \in G-\operatorname{co}(A)=\Lambda(A)$, such that $\bar{y} \notin \bigcup_{i=1}^{n} F\left(x_{i}\right)$. Thus $f\left(x_{i}, \bar{y}\right)+h(\bar{y})-$ $h\left(x_{i}\right)>0$ for $i=1, \ldots, n$. Therefore,

$$
\min _{1 \leq i \leq n}\left[f\left(x_{i}, \bar{y}\right)+h(\bar{y})-h\left(x_{i}\right)\right]>0
$$

which contradicts the assumption (b). Hence $F: X \rightarrow 2^{X}$ is a G-KKM map. Moreover we have

(i) $\quad F\left(x_{0}\right) \subset K$ by $(d)$, so that $c_{X} F\left(x_{0}\right) \subset c l_{X} K=K$ and hence $c l_{X} F\left(x_{0}\right)$ is compact in $X$;

(ii) for each $A \in\langle X\rangle$ with $x_{0} \in A$ and each $x \in G$-co $(A)$,

$$
\begin{aligned}
F(x) \cap G-c o(A) & =\{y \in G-c o(A): f(x, y)+h(y)-h(x) \leq 0\} \\
& =\{y \in G-c o(A): f(x, y)+h(y) \leq h(x)\}
\end{aligned}
$$

is closed in $G-\operatorname{co}(A)$ by $(a)$ and the fact that $h$ is lower semicontinuous on $G$ $\operatorname{co}(A)$

(iii) for each $A \in\langle X\rangle$ with $x_{0} \in A$, if $y \in\left(c_{X}\left(\bigcap_{x \in G-c o(A)} F(x)\right)\right) \cap G-c o(A)$, then $y \in G-\operatorname{co}(A)$ and there is a net $\left\{y_{\alpha}\right\}_{\alpha \in \Gamma}$ in $\bigcap_{x \in G-c o(A)} F(x)$ such that $y_{\alpha} \rightarrow y$. For each $x \in G-c o(A)$, since $G-c o(\{x, y\}) \subset G-c o(A)$, we have $y_{\alpha} \in F(z)$ for all $\alpha \in \Gamma$ and all $z \in G-c o(\{x, y\})$. This implies that $f\left(z, y_{\alpha}\right)+h\left(y_{\alpha}\right)-h(z) \leq 0$ for all $\alpha \in \Gamma$ and all $z \in G-c o(\{x, y\})$ so that by $(c), \quad f(x, y)+h(y)-h(\bar{x}) \leq 0$; it follows that $y \in\left(\bigcap_{x \in G-c o(A)} F(x)\right) \cap G$ co $(A)$. Hence, $\quad\left(c_{X}\left(\bigcap_{x \in G-c o(A)} F(x)\right)\right) \cap G-\operatorname{co}(A)=\left(\bigcap_{x \in G-\operatorname{co}(A)} F(x)\right)$ $\cap G-\operatorname{co}(A)$.

Hence by Theorem 2.1 we have $\bigcap_{x \in X} F(x) \neq \emptyset$. Then there exists $\widehat{y} \in$ $\bigcap_{x \in X} F(x)$, so that $f(x, \widehat{y})+h(\widehat{y})-h(x) \leq 0$ for all $x \in X$, i.e., $f(x, \widehat{y}) \leq h(x)-h(\widehat{y})$ for all $x \in X$.

Theorem 3.1 generalizes Theorem 1 of Chowdhury and Tan in [5] into G-convex spaces.

When $h \equiv 0$, Theorem 3.1 reduces to the following.

Theorem 3.2: Let $(E, \Gamma)$ be a $G$-convex space such that, for each $x \in E,\{x\}$ is $G$ convex (i.e., $\Gamma(\{x\})=\{x\})$, and for each $A \in\langle E\rangle, G-c o(A)$ is compact and $\Gamma(A)=$ $G$-co $(A)$. Let $X$ be a non-empty $G$-convex subset of $E$. Let $f: X \times X \rightarrow \mathbb{R} \cup$ $\{-\infty,+\infty\}$ be such that

(a) for each $A \in\langle X\rangle$ and each fixed $x \in G-c o(A), y \mapsto f(x, y)$ is lower semicontinuous on $G-\operatorname{co}(A)$;

(b) for each $A \in\langle X\rangle$ and each $y \in G-\operatorname{co}(A), \min _{x \in A} f(x, y) \leq 0$;

(c) for each $A \in\langle X\rangle$ and each $x, y \in G-\operatorname{co}(A)$ and every net $\left\{y_{\alpha}\right\}_{\alpha \in \Gamma}$ in $X$ converging to $y$ with $f\left(z, y_{\alpha}\right) \leq 0$ for all $\alpha \in \Gamma$ and all $z \in G-c o(\{x, y\})$, we have $f(x, y) \leq 0$;

(d) there exist a non-empty closed and compact subset $K$ of $X$ and $x_{0} \in K$ such that $f\left(x_{0}, y\right)>0$ for all $y \in X \backslash K$. 
Then there exists $\widehat{y} \in K$ such that $f(x, \widehat{y}) \leq 0$ for all $x \in X$.

Theorem 3.2 generalizes Theorem 2 of Chowdhury and Tan in [5] into G-convex spaces.

Note that Theorem 3.2 implicitly implies the following minimax inequality.

Theorem 3.3: Let $(E, \Gamma)$ be a $G$-convex space such that, for each $x \in E,\{x\}$ is $G$ convex (i.e., $\Gamma(\{x\})=\{x\}$ ), and for each $A \in\langle E\rangle, G-c o(A)$ is compact and $\Gamma(A)=$ $G$-co $(A)$. Let $X$ be a non-empty $G$-convex subset of $E$. Let $f: X \times X \rightarrow \mathbb{R} \cup$ $\{-\infty,+\infty\}$ be such that

(a) for each $A \in\langle X\rangle$ and each fixed $x \in G-c o(A), y \mapsto f(x, y)$ is lower semicontinuous on $G$-co $(A)$;

(b) for each $A \in\langle X\rangle$ and each $y \in G-c o(A), \min _{x \in A} f(x, y) \leq 0$;

(c) for each $A \in\langle X\rangle$ and each $x, y \in G-c o(A)$ and every net $\left\{y_{\alpha}\right\}_{\alpha \in \Gamma}$ in $X$ converging to $y$ with $f\left(z, y_{\alpha}\right) \leq 0$ for all $\alpha \in \Gamma$ and all $z \in G-c o(\{x, y\})$, we have $f(x, y) \leq 0$;

(d) there exist a non-empty closed and compact subset $K$ of $X$ and $x_{0} \in K$ such that, whenever $\sup _{x \in X} f(x, x)<\infty, \quad f\left(x_{0}, y\right)>\sup _{x \in X} f(x, x)$ for all $y \in X \backslash K$.

Then the minimax inequality,

holds.

$$
\min _{y \in K} \sup _{x \in X} f(x, y) \leq \sup _{x \in X} f(x, x),
$$

Proof: Let $t=\sup _{x \in X} f(x, x)$. Clearly, we may assume that $t<+\infty$. Define for any $x, y \in X, g(x, y)=f(x, y)-t$. Then $g$ satisfies all the hypotheses of Theorem 3.2 when $f$ is replaced by $g$. Hence, by Theorem 3.2 , there exists a $\widehat{y} \in K$ such that $g(x, \widehat{y}) \leq 0$ for all $x \in X$. This implies $f(x, \widehat{y}) \leq t$ for all $x \in X$, so that $\sup _{x \in X} f(x, \widehat{y}) \leq t$ and, therefore,

i.e.,

$$
\begin{gathered}
\min _{y \in K} \sup _{x \in X} f(x, y) \leq \sup _{x \in X} f(x, \widehat{y}) \leq t=\sup _{x \in X} f(x, x), \\
\min _{y \in K} \sup _{x \in X} f(x, y) \leq \sup _{x \in X} f(x, x) .
\end{gathered}
$$

Theorem 3.3 generalizes Theorem 3 of Chowdhury and Tan in [5] into G-convex spaces. Theorem 3.3 also generalizes Theorem 1 in [7] in several ways.

Theorem 3.4: Let $(E, \Gamma)$ be a $G$-convex space such that, for each $x \in E,\{x\}$ is $G$ convex (i.e., $\Gamma(\{x\})=\{x\}$ ), and for each $A \in\langle E\rangle, G-c o(A)$ is compact and $\Gamma(A)=$ $G$-co $(A)$. Let $X$ be a non-empty $G$-convex subset of $E$ and let $f, g: X \times X \rightarrow \mathbb{R} \cup$ $\{-\infty,+\infty\}$ be such that

(a) $f(x, y) \leq g(x, y)$ for all $x, y \in X$ and $g(x, x) \leq 0$ for all $x \in X$;

(b) for each $A \in\langle X\rangle$ and each fixed $x \in G-\operatorname{co}(A), y \mapsto f(x, y)$ is lower semicontinuous on $G-c o(A)$;

(c) for each $y \in X$, the set $\{x \in X: g(x, y)>0\}$ is $G$-convex;

(d) for each $A \in\langle X\rangle$ and each $x, y \in G$-co $(A)$ and every net $\left\{y_{\alpha}\right\}_{\alpha \in \Gamma}$ in $X$ converging to $y$ with $f\left(z, y_{\alpha}\right) \leq 0$ for all $\alpha \in \Gamma$ and all $z \in G-c o(\{x, y\})$, we have $f(x, y) \leq 0$;

(e) there exist a non-empty closed and compact subset $K$ of $X$ and $x_{0} \in K$ such that $f\left(x_{0}, y\right)>0$ for all $y \in X \backslash K$.

Then there exists $\widehat{y} \in K$ such that $f(x, \widehat{y}) \leq 0$ for all $x \in X$.

Proof: It is easy to see that the conditions $(a)$ and $(c)$ here imply the condition $(b)$ of Theorem 3.2, so that the conclusions follows.

Note that Theorem 3.4 generalizes Theorem 4 of Chowdhury and Tan in [5] into 
$G$-convex spaces and, in turn, generalizes Theorem 1 of Shih and Tan in [13, pp. 280$282]$.

Theorem 3.5: Let $(E, \Gamma)$ be a $G$-convex space such that, for each $x \in E,\{x\}$ is $G$ convex (i.e., $\Gamma(\{x\})=\{x\}$ ), and for each $A \in\langle E\rangle, G-c o(A)$ is compact and $\Gamma(A)=$ $G$-co $(A)$. Let $C$ be a non-empty closed and $G$-convex subset of $E$ and let $f: C \times C \rightarrow \mathbb{R}$ be such that

(a) $f(x, x) \leq 0$ for all $x \in C$;

(b) for each $A \in\langle C\rangle$ and each fixed $x \in G-c o(A), y \mapsto f(x, y)$ is lower semicontinuous on $G$-co $(A)$;

(c) for each $y \in C$, the set $\{x \in C: f(x, y)>0\}$ is $G$-convex;

(d) for each $A \in\langle C\rangle$ and each $x, y \in G-c o(A)$ and every net $\left\{y_{\alpha}\right\}_{\alpha} \in \Gamma$ in $C$ converging to $y$ with $f\left(z, y_{\alpha}\right) \leq 0$ for all $\alpha \in \Gamma$ and all $z \in G-\operatorname{co}(\{x, y\})$, we have $f(x, y) \leq 0$;

(e) there exist a non-empty closed and compact subset $L$ of $E$ and $x_{0} \in C \cap L$ such that $f\left(x_{0}, y\right)>0$ for all $y \in C \backslash L$.

Then there exists $\widehat{y} \in C \cap L$ such that $f(x, \widehat{y}) \leq 0$ for all $x \in C$.

Proof: Let $f=g, K=C \cap L$ and $X=C$ in Theorem 3.4. The conclusion follows.

Theorem 3.5 generalizes Theorem 5 of Chowdhury and Tan in [5] into G-convex spaces and, in turn, improves and generalizes Theorem 1 of Brézis-Nirenburg-Stampacchia in [4]. Note that if the compact set $L$ is a subset of $C, C$ is not required to be closed in $E$ in Theorem 3.5. Note also that in Theorem 1 of [4], the set $C$ was not assumed to be closed in $E$. However, this is false in general as is observed by the Example 1.3.14 in [14].

It is important to be noted here that, as applications to Theorem 3.2 , we may obtain results on existence theorems of equilibria in $G$-convex spaces. The author plans to continue work on this topic in the near future.

\section{Equivalent Formulations of Theorem 3.2}

Following the ideas of Ky Fan [7, pp. 103-113], Ding and Tan [6, pp. 237-239] and Tan and Yuan [15, pp. 486-489], we shall obtain several equivalent formulations of Theorem 3.2 and fixed point theorems. For ready reference, we shall now state and prove four equivalent formulations of Theorem 3.2 and four fixed point theorems.

Theorem 3.2-A: (First Geometric Form) Let $(E, \Gamma)$ be a G-convex space such that, for each $x \in E,\{x\}$ is $G$-convex (i.e., $\Gamma(\{x\})=\{x\}$ ), and for each $A \in\langle E\rangle, G$ $\operatorname{co}(A)$ is compact and $\Gamma(A)=G$-co $(A)$. Suppose that $X$ is a non-empty $G$-convex subset of $E$ and $N \subset X \times X$ is such that

$\left(a_{1}\right)$ for each $A \in\langle X\rangle$ and each fixed $x \in G-\operatorname{co}(A)$, the set $\{y \in G-\operatorname{co}(A)$ : $(x, y) \in N\}$ is open in $G-\operatorname{co}(A)$;

$\left(b_{1}\right)$ for each $A \in\langle X\rangle$ and each $y \in G-c o(A)$, there exists $x \in A$ such that $(x, y) \notin N$;

$\left(c_{1}\right)$ for each $A \in\langle X\rangle$ and each $x, y \in G-c o(A)$ and every net $\left\{y_{\alpha}\right\}_{\alpha \in \Gamma}$ in $X$ converging to $y$ such that $\left(z, y_{\alpha}\right) \notin N$ for all $\alpha \in \Gamma$ and for all $z \in G-c o(\{x, y\})$, we have $(x, y) \notin N$;

$\left(d_{1}\right)$ there exist a non-empty closed and compact subset $K$ of $X$ and $x_{0} \in K$ such that $\left(x_{0}, y\right) \in N$, for all $y \in X \backslash K$.

Then there exists $\widehat{y} \in K$ such that the set $\{x \in X:(x, \widehat{y}) \in N\}=\emptyset$. 
Theorem 3.2-B: (Second Geometric Form) Let $(E, \Gamma)$ be a $G$-convex space such that, for each $x \in E,\{x\}$ is $G$-convex (i.e., $\Gamma(\{x\})=\{x\})$, and for each $A \in\langle E\rangle, G$ $c o(A)$ is compact and $\Gamma(A)=G-c o(A)$. Suppose that $X$ is a non-empty $G$-convex subset of $E$ and $M \subset X \times X$ is such that

$\left(a_{2}\right)$ for each $A \in\langle X\rangle$ and each fixed $x \in G-c o(A)$, the set $\{y \in G-\operatorname{co}(A)$ : $(x, y) \in M\}$ is closed in $G-\operatorname{co}(A)$;

$\left(b_{2}\right)$ for each $A \in\langle X\rangle$ and each $y \in G-c o(A)$, there exists $x \in A$ such that $(x, y) \in M$

$\left(c_{2}\right)$ for each $A \in\langle X\rangle$ and each $x, y \in G-c o(A)$ and every net $\left\{y_{\alpha}\right\}_{\alpha \in \Gamma}$ in $X$ converging to $y$ such that $\left(z, y_{\alpha}\right) \in M$ for all $\alpha \in \Gamma$ and for all $z \in G-\operatorname{co}(\{x, y\})$, we have $(x, y) \in M$;

$\left(d_{2}\right)$ there exist a non-empty closed and compact subset $K$ of $X$ and $x_{0} \in K$ such that $\left(x_{0}, y\right) \notin M$, for all $y \in X \backslash K$.

Then there exists a point $\widehat{y} \in K$ such that $X \times\{\widehat{y}\} \subset M$.

Theorem 3.2-C: (Maximal Element Version) Let $(E, \Gamma)$ be a $G$-convex space such that, for each $x \in E,\{x\}$ is $G$-convex (i.e., $\Gamma(\{x\})=\{x\})$, and for each $A \in\langle E\rangle$, G$c o(A)$ is compact and $\Gamma(A)=G-c o(A)$. Suppose that $X$ is a non-empty $G$-convex subset of $E$ and $G: X \rightarrow 2^{X}$ is a set-valued map such that

$\left(a_{3}\right)$ for each $A \in\langle X\rangle$ and each fixed $x \in G-\operatorname{co}(A), G^{-1}(x) \cap G-c o(A)=\{y \in G-$ $\operatorname{co}(A): x \in G(y)\}$ is open in $G-c o(A)$;

$\left(b_{3}\right)$ for each $A \in\langle X\rangle$ and each $y \in G-c o(A)$, there exists $x \in A$ such that $x \notin G(y)$;

$\left(c_{3}\right)$ for each $A \in\langle X\rangle$ and each $x, y \in G-c o(A)$ and every net $\left\{y_{\alpha}\right\}_{\alpha \in \Gamma}$ in $X$ converging to $y$ such that $z \notin G\left(y_{\alpha}\right)$, for all $\alpha \in \Gamma$ and for all $z \in G-c o(\{x, y\})$, we have $x \notin G(y)$;

$\left(d_{3}\right)$ there exist a non-empty closed and compact subset $K$ of $X$ and $x_{0} \in K$ such that $x_{0} \in G(y)$, for all $y \in X \backslash K$.

Then there exists a point $\widehat{y} \in K$ such that $G(\widehat{y})=\emptyset$.

Theorem 3.2-D: (Fixed Point Version) Let $(E, \Gamma)$ be a $G$-convex space such that, for each $x \in E,\{x\}$ is $G$-convex (i.e., $\Gamma(\{x\})=\{x\})$, and for each $A \in\langle E\rangle, G$-co $(A)$ is compact and $\Gamma(A)=G-c o(A)$. Suppose that $X$ is a non-empty $G$-convex subset of $E$ and $G: X \rightarrow 2^{X}$ is a set-valued map such that

$\left(a_{4}\right)$ for each $A \in\langle X\rangle$ and each fixed $x \in G-\operatorname{co}(A), G^{-1}(x) \cap G-c o(A)$ is open in $G-\operatorname{co}(A)$

$\left(b_{4}\right)$ for each $A \in\langle X\rangle$ and each $x, y \in G$-co $(A)$ and every net $\left\{y_{\alpha}\right\}_{\alpha \in \Gamma}$ in $X$ converging to $y$ such that $z \notin G\left(y_{\alpha}\right)$, for all $\alpha \in \Gamma$ and for all $z \in G-c o(\{x, y\})$, we have $x \notin G(y)$;

$\left(c_{4}\right)$ there exist a non-empty closed and compact subset $K$ of $X$ and $x_{0} \in K$ such that $x_{0} \in G(y)$, for all $y \in X \backslash K$;

$\left(d_{4}\right)$ for each $y \in K, G(y) \neq \emptyset$.

Then there exists $y_{0} \in X$ such that $y_{0} \in G-\operatorname{co}\left(G\left(y_{0}\right)\right)$.

Theorem 3.2-D implies the following fixed point theorem.

Theorem 3.6-A: (Second Fixed Point Theorem) Let $(E, \Gamma)$ be a G-convex space such that, for each $x \in E,\{x\}$ is G-convex (i.e., $\Gamma(\{x\})=\{x\}$ ), and for each $A \in\langle E\rangle, G-c o(A)$ is compact and $\Gamma(A)=G-c o(A)$. Suppose that $X$ is a non-empty $G$-convex subset of $E$ and $G: X \rightarrow 2^{X}$ is a set-valued map such that

$\left(a_{5}\right)$ for each $A \in\langle X\rangle$ and each fixed $x \in G-\operatorname{co}(A), G^{-1}(x) \cap G-c o(A)$ is open in $G-\operatorname{co}(A)$;

$\left(b_{5}\right)$ for each $y \in X, G(y)$ is $G$-convex; 
$\left(c_{5}\right)$ for each $A \in\langle X\rangle$ and $x, y \in G-c o(A)$ and every net $\left\{y_{\alpha}\right\}_{\alpha \in \Gamma}$ in $X$ converging to $y$ such that $z \notin G\left(y_{\alpha}\right)$, for all $\alpha \in \Gamma$ and for all $z \in G-\operatorname{co}(\{x, y\})$, we have $x \notin G(y)$;

$\left(d_{5}\right)$ there exist a non-empty closed and compact subset $K$ of $X$ and $x_{0} \in K$ such that $x_{0} \in G(y)$, for all $y \in X \backslash K$;

$\left(e_{5}\right)$ for each $y \in K, G(y) \neq \emptyset$.

Then there exists a point $y_{0} \in X$ such that $y_{0} \in G\left(y_{0}\right)$.

The following fixed point theorem is equivalent to Theorem 3.6-A.

Theorem 3.6-B: (Third Fixed Point Theorem) Let $(E, \Gamma)$ be a G-convex space such that, for each $x \in E,\{x\}$ is G-convex (i.e., $\Gamma(\{x\})=\{x\})$, and for each $A \in$ $\langle E\rangle, G-c o(A)$ is compact and $\Gamma(A)=G-c o(A)$. Suppose that $X$ is a non-empty $G$ convex subset of $E$ and $Q: X \rightarrow 2^{X}$ is a set-valued map such that

$\left(a_{6}\right)$ for each $A \in\langle X\rangle$ and each fixed $x \in G-\operatorname{co}(A), Q(x) \cap G-\operatorname{co}(A)$ is open in $G$ $\operatorname{co}(A)$;

$\left(b_{6}\right)$ for each $y \in X, Q^{-1}(y)$ is $G$-convex;

$\left(c_{6}\right)$ for each $a \in\langle X\rangle$ and each $x, y \in G-c o(A)$ and every net $\left\{y_{\alpha}\right\}_{\alpha \in \Gamma}$ in $X$ converging to $y$ such that $z \notin Q^{-1}\left(y_{\alpha}\right)$, for all $\alpha \in \Gamma$ and for all $z \in G-\operatorname{co}(\{x, y\})$, we have $x \notin Q^{-1}(y)$;

$\left(d_{6}\right)$ there exist a non-empty closed and compact subset $K$ of $X$ and $x_{0} \in K$ such that $x_{0} \in Q^{-1}(y)$, for all $y \in X \backslash K$;

$\left(e_{6}\right)$ for each $y \in K, Q^{-1}(y) \neq \emptyset$.

Then there exists a point $y_{0} \in X$ such that $y_{0} \in Q\left(y_{0}\right)$.

The following fixed point theorem follows from Theorem 3.6-A.

Theorem 3.7: (Fourth Fixed Point Theorem) Let $(E, \Gamma)$ be a $G$-convex space such that, for each $x \in E,\{x\}$ is $G$-convex (i.e., $\Gamma(\{x\})=\{x\})$, and for each $A \in\langle E\rangle, G$ $c o(A)$ is compact and $\Gamma(A)=G-c o(A)$. Suppose that $X$ is a non-empty G-convex subset of $E$ and $G: X \rightarrow 2^{X}$ is a set-valued map such that

$\left(a_{7}\right)$ for each $A \in\langle X\rangle$ and each fixed $x \in G-\operatorname{co}(A), G^{-1}(X) \cap G-\operatorname{co}(A)$ is open in $G$-co $(A)$;

$\left(b_{7}\right)$ for each $A \in\langle X\rangle$ and each $x, y \in G-c o(A)$ and every net $\left\{y_{\alpha}\right\}_{\alpha \in \Gamma}$ in $X$ converging to $y$ such that $z \notin G-\operatorname{co}\left(G\left(y_{\alpha}\right)\right)$, for all $\alpha \in \Gamma$ and for all $z \in G$ $\operatorname{co}(\{x, y\})$, we have $x \notin G-c o(G(y))$;

$\left(c_{7}\right)$ there exist a non-empty closed and compact subset $K$ of $X$ and $x_{0} \in K$ such that $x_{0} \in G-c o(G(y))$, for all $y \in X \backslash K$;

$\left(d_{7}\right)$ for each $y \in K, G(y) \neq \emptyset$.

Then there exists $y_{0} \in X$ such that $y_{0} \in G-\operatorname{co}\left(G\left(y_{0}\right)\right)$.

Proof Theorem 3.2 $\Rightarrow$ Theorem 3.2-A: Let $f: X \times X \rightarrow \mathbb{R}$ be such that

Then we have the following.

$$
f(x, y)= \begin{cases}1, & \text { if }(x, y) \in N \\ 0, & \text { if }(x, y) \notin N .\end{cases}
$$

(a) For each $\lambda \in \mathbb{R}$, each $A \in\langle X\rangle$ and each fixed $x \in G$-co $(A)$, the set

$$
\{y \in G-\operatorname{co}(A): f(x, y) \leq \lambda\}= \begin{cases}\emptyset, & \text { if } \lambda<0 \\ \{y \in G-\operatorname{co}(A):(x, y) \notin N\}, & \text { if } 0 \leq \lambda<1 \\ G-\operatorname{co}(A), & \text { if } \lambda \geq 1\end{cases}
$$

is closed in $G-\operatorname{co}(A)$. Thus for each $A \in\langle X\rangle$ and each fixed $x \in G-\operatorname{co}(A)$, 
$y \mapsto f(x, y)$ is lower semicontinuous on $G$-co $(A)$.

(b) For each $A \in\langle X\rangle$ and each $y \in G-c o(A)$, there exists $x \in A$ such that $(x, y) \notin N$. Thus $f(x, y)=0$. Hence $\min _{x \in A} f(x, y) \leq 0$.

(c) By hypothesis $\left(c_{1}\right)$, for each $A \in\langle X\rangle$ and each $x, y \in G$-co $(A)$ and every net $\left\{y_{\alpha}\right\}_{\alpha \in \Gamma}$ in $X$ converging to $y$ such that $f\left(z, y_{\alpha}\right) \leq 0$ for all $\alpha \in \Gamma$ and for all $z \in G-c o(\{x, y\})$, we have $f(x, y) \leq 0$.

(d) There exist a non-empty closed and compact subset $K$ of $X$ and $x_{0} \in K$ such that $\left(x_{0}, y\right) \in N$, i.e., $f\left(x_{0}, y\right)>0$ for all $y \in X \backslash K$.

Hence, all the hypotheses of Theorem 3.2 are satisfied. Therefore, by Theorem 3.2, there exists $\widehat{y} \in K$ such that $f(x, \widehat{y}) \leq 0$ for all $x \in X$; i.e., there exists $\widehat{y} \in K$ such that $\{x \in X:(x, \widehat{y}) \in N\}=\emptyset$.

Proof Theorem 3.2-A $\Rightarrow$ Theorem 3.2: Let $N=\{(x, y) \in X \times X: f(x, y)>0\}$. Then we have the following.

$\left(a_{1}\right)$ For each $A \in\langle X\rangle$ and each fixed $x \in G-\operatorname{co}(A)$, the set $\{y \in G-\operatorname{co}(A)$ : $f(x, y)>0\}$ is open in $G-c o(A)$. Hence the set $\{y \in G-c o(A):(x, y) \in N\}$ is open in $G-c o(A)$.

$\left(b_{1}\right)$ For each $A \in\langle X\rangle$ and each $y \in G-c o(A), \min _{x \in A} f(x, y) \leq 0$. Thus there exists $x \in A$ such that $f(x, y) \leq 0$, i.e., $(x, y) \notin N$.

$\left(c_{1}\right)$ By condition $(c)$, for each $A \in\langle X\rangle$ and each $x, y \in G-c o(A)$ and every net $\left\{y_{\alpha}\right\}_{\alpha \in \Gamma}$ in $X$ converging to $y$ such that $\left(z, y_{\alpha}\right) \notin N$ for all $\alpha \in \Gamma$ and for all $z \in G$-co $(\{x, y\})$, we have $(x, y) \notin N$.

$\left(d_{1}\right)$ By condition $(d)$, there exist a non-empty closed and compact subset $K$ of $X$ and $x_{0} \in K$ such that $\left(x_{0}, y\right) \in N$ for all $y \in X \backslash K$.

Hence, all the hypotheses of Theorem 3.2-A are satisfied. Therefore, by Theorem 3.2 -A, there exists $\widehat{y} \in K$ such that $\{x \in X:(x, \widehat{y}) \in N\}=\emptyset$. Thus $(x, \widehat{y}) \notin N$ for all $x \in X$. Hence, $f(x, \widehat{y}) \leq 0$ for all $x \in X$.

Proof Theorem 3.2-A $\Rightarrow$ Theorem 3.2-B: Let $N=X \times X \backslash M$. Then we have the following.

$\left(a_{1}\right)$ For each $A \in\langle X\rangle$ and each fixed $x \in G-c o(A)$, the set $\{y \in G-c o(A)$ : $(x, y) \in N\}$ is open in $G-\operatorname{co}(A)$.

$\left(b_{1}\right)$ For each $A \in\langle X\rangle$ and each $y \in G-c o(A)$, there exists $x \in A$ such that $(x, y) \notin N$.

$\left(c_{1}\right)$ For each $A \in\langle X\rangle$ and each $x, y \in G-c o(A)$ and every net $\left\{y_{\alpha}\right\}_{\alpha \in \Gamma}$ in $X$ converging to $y$ such that $\left(z, y_{\alpha}\right) \notin N$ for all $\alpha \in \Gamma$ and for all $z \in G$-co $(\{x, y\})$, we have $(x, y) \notin N$.

$\left(d_{1}\right)$ There exist a non-empty closed and compact subset $K$ of $X$ and $x_{0} \in K$ such that $\left(x_{0}, y\right) \in N$, for all $y \in X \backslash K$.

Hence, all the hypotheses of Theorem 3.2-A are satisfied. Therefore, by Theorem 3.2 -A, there exists $\widehat{y} \in K$ such that $(x, \widehat{y}) \notin N$ for all $x \in X$. Thus $(x, \widehat{y}) \in M$ for all $x \in X$. Hence $X \times\{\widehat{y}\} \subset M$.

Proof Theorem 3.2-B $\Rightarrow$ Theorem 3.2-A: Let $M=X \times X \backslash N$. Then the proof is similar to the above proof and, therefore, by Theorem 3.2-B, there exists $\widehat{y} \in K$ such that $X \times\{\widehat{y}\} \subset M$. Hence, $(x, \widehat{y}) \notin N$ for all $x \in X$, i.e., the set $\{x \in X:(x, \widehat{y}) \in$ $N\}=\emptyset$.

Proof Theorem 3.2-B $\Rightarrow$ Theorem 3.2-C: Let $M=\{(x, y) \in X \times X: x \in G(y)\}$. Then we have the following.

$\left(a_{2}\right)$ For each $A \in\langle X\rangle$ and each fixed $x \in G-c o(A)$, the set $\{y \in G-c o(A): x \notin$ $G(y)\}=\{y \in G-c o(A):(x, y) \in M\}$ is closed in $G-c o(A)$.

$\left(b_{2}\right)$ For each $A \in\langle X\rangle$ and each $y \in G$-co $(A)$, there exists $x \in A$ such that $x \notin$ 
$G(y)$ so that $(x, y) \in M$.

$\left(c_{2}\right)$ By condition $\left(c_{3}\right)$, for each $A \in\langle X\rangle$ and each $x, y \in G-c o(A)$ and every net $\left\{y_{\alpha}\right\}_{\alpha \in \Gamma}$ in $X$ converging to $y$ such that $\left(z, y_{\alpha}\right) \in M$ for all $\alpha \in \Gamma$ and for all $z \in G-c o(\{x, y\})$, we have $(x, y) \in M$.

$\left(d_{2}\right)$ By condition $\left(d_{3}\right)$, there exist a non-empty closed and compact subset $K$ of $X$ and $x_{0} \in K$ such that $\left(x_{0}, y\right) \notin M$ for all $y \in X \backslash K$.

Hence, all the hypotheses of Theorem 3.2-B are satisfied. Therefore, by Theorem $3.2-\mathrm{B}$, there exists $\widehat{y} \in K$ such that $X \times\{\widehat{y}\} \subset M$. Thus $x \notin G(\widehat{y})$ for all $x \in X$. Hence $G(\widehat{y})=\emptyset$.

Proof Theorem 3.2-C $\Rightarrow$ Theorem 3.2-B: Let $G: X \rightarrow 2^{X}$ be defined by $G(y)=$ $\{x \in X:(x, y) \notin M\}$ for all $y \in X$. Then we have the following.

$\left(a_{3}\right)$ For each $A \in\langle X\rangle$ and each fixed $x \in G-c o(A)$, the set $\{y \in G-c o(A):(x, y) \notin$ $M\}=\{y \in G-c o(A): x \in G(y)\}$ is open in $G-c o(A)$.

$\left(b_{3}\right)$ For each $A \in\langle X\rangle$ and each $y \in G-c o(A)$, there exists $x \in A$ such that $(x, y) \in M$ so that $x \notin G(y)$.

$\left(c_{3}\right)$ By condition $\left(c_{2}\right)$, for each $A \in\langle X\rangle$ and each $x, y \in G-c o(A)$ and every net $\left\{y_{\alpha}\right\}_{\alpha \in \Gamma}$ in $X$ converging to $y$ such that $z \notin G\left(y_{\alpha}\right)$ for all $\alpha \in \Gamma$ and for all $z \in G$-co $(\{x, y\})$, we have $x \notin G(y)$.

$\left(d_{3}\right)$ There exist a non-empty closed and compact subset $K$ of $X$ and $x_{0} \in K$ such that $\left(x_{0}, y\right) \notin M$ for all $y \in X \backslash K$, so that $x_{0} \in G(y)$ for all $y \in X \backslash K$.

Hence, all the hypotheses of Theorem 3.2-C are satisfied. Therefore, by Theorem $3.2-\mathrm{C}$, there exists a point $\widehat{y} \in K$ such that $G(\widehat{y})=\emptyset$. Thus $x \notin G(\widehat{y})$ for all $x \in X$. Hence, $(x, \widehat{y}) \in M$ for all $x \in X$, i.e., $X \times\{\widehat{y}\} \subset M$.

Proof Theorem 3.2-C $\Rightarrow$ Theorem 3.2-D: By Theorem 3.2-C, there exist $A \in\langle X\rangle$ and $y_{0} \in G-\operatorname{co}(A)$ such that $x \in G\left(y_{0}\right)$ for all $x \in A$. Thus $y_{0} \in G-\operatorname{co}(A) \subset G$ $\operatorname{co}\left(G\left(y_{0}\right)\right)$.

Proof Theorem 3.2-D $\Rightarrow$ Theorem 3.2-C: From the hypotheses of Theorem 3.2-C, we see that the conditions $\left(a_{4}\right),\left(b_{4}\right)$ and $\left(c_{4}\right)$ follow from the conditions $\left(a_{3}\right),\left(c_{3}\right)$ and $\left(d_{3}\right)$ respectively. Suppose for each $y \in K, G(y) \neq \emptyset$. Then condition $\left(d_{4}\right)$ of Theorem 3.2-D is satisfied. Hence, all the hypotheses of Theorem 3.2-D are satisfied. Therefore, by Theorem 3.2-D, there exists $y_{0} \in X$ such that $y_{0} \in G-c o\left(G\left(y_{0}\right)\right)$. Thus, by Lemma A, there exist $A=\left\{x_{1}, \ldots, x_{n}\right\} \in\left\langle G\left(y_{0}\right)\right\rangle$ such that $y_{0} \in G-c o(A)$. Now, $A \in\langle X\rangle$ and $y_{0} \in G-c o(A)$. Hence, by condition $\left(b_{3}\right)$ of Theorem 3.2-C, there exists $x_{i} \in A$ such that $x_{i} \notin G\left(y_{0}\right)$, which is a contradiction. Hence, there exists $\widehat{y} \in K$ such that $G(\widehat{y})=\emptyset$.

Proof Theorem 3.2-D $\Rightarrow$ Theorem 3.6-A: This is obvious, because by Theorem 3.2D there exists $y_{0} \in X$ such that $y_{0} \in G-c o\left(G\left(y_{0}\right)\right)$. But by $\left(b_{5}\right)$ of Theorem 3.6-A, $G\left(y_{0}\right)$ is $G$-convex. Hence $y_{0} \in G\left(y_{0}\right)$.

Proof Theorem 3.6-A $\Leftrightarrow$ Theorem 3.6-B:

$\left(\Rightarrow:\right.$ ) Let $G=Q^{-1}$; then $G^{-1}=Q$. Therefore, by Theorem 3.6-A, there exists $y_{0} \in X$ such that $y_{0} \in G\left(y_{0}\right)=Q^{-1}\left(y_{0}\right)$. Hence $y_{0} \in Q\left(y_{0}\right)$.

$(\Leftarrow:)$ Let $Q=G^{-1}$; then $Q^{-1}=G$. Therefore, by Theorem 3.6-B, there exists $y_{0} \in X$ such that $y_{0} \in Q\left(y_{0}\right)=G^{-1}\left(y_{0}\right)$. Hence $y_{0} \in G\left(y_{0}\right)$.

Proof Theorem 3.6-A $\Rightarrow$ Theorem 3.7: Let $F(y)=G$-coG(y). Then we have the following.

$\left(a_{5}\right)$ For each $A \in\langle X\rangle$ and each fixed $x \in G-c o(A), F^{-1}(x) \cap G-c o(A)$ is open in $G-\operatorname{co}(A)$. Let $y \in(G-c o G)^{-1}(x) \cap G-c o(A)$. Then $y \in G-c o(A)$ and $x \in G$ $c o(G(y))$. Thus, by Lemma A, there exists $C=\left\{y_{1}, \ldots, y_{n}\right\} \in\langle G(y)\rangle$ such 
that $x \in G-\operatorname{co}(C)$. Now, for each $i=1, \ldots, n, G^{-1}\left(y_{i}\right) \cap G-\operatorname{co}(A)$ is open in $G$-co $(A)$; and $y \in G^{-1}\left(y_{i}\right) \cap G-c o(A)$ for all $i$. Let $U=\bigcap_{i=1}^{n}\left(G^{-1}\left(y_{i}\right) \cap G\right.$ $\operatorname{co}(A))$. Then $U$ is an open neighborhood of $y$ in $G-\operatorname{co}(A)$. If $z \in U$, then $z \in G-\operatorname{co}(A)$ and $y_{i} \in G(z)$ for all $i=1, \ldots, n$. So $x \in G-\operatorname{co}(C) \subset G$ $c o(G(z))=F(z)$ and, hence, $z \in(G-c o G)^{-1}(x) \cap G-c o(A)=F^{-1}(x) \cap G$ $\operatorname{co}(A)$ for all $z \in U$. Hence $y \in U \subset F^{-1}(x) \cap G-c o(A)$. Therefore, $F^{-1}(x) \cap G-c o(A)$ is open in $G-c o(A)$.

$\left(b_{5}\right)$ For each $y \in X, F(y)=G-c o(G(y))$ is $G$-convex.

Conditions $\left(c_{5}\right)$ and $\left(d_{5}\right)$ are obvious. But for each $y \in K, G(y) \neq \emptyset$ implies $F(y) \neq \emptyset$. Thus $\left(e_{5}\right)$ holds.

Therefore, by Theorem 3.6-A, there exists $y_{0} \in X$ such that $y_{0} \in F\left(y_{0}\right)=G$ $\operatorname{co}\left(G\left(y_{0}\right)\right)$.

\section{References}

[1] Bardaro, C. and Ceppitelli, R., Some further generalizations of Knaster-Kuratowski-Mazurkiewicz theorem and minimax inequalities, J. Math. Anal. Appl. 132 (1988), 484-490.

[2] Bardaro, C. and Ceppitelli, R., Applications to the generalized Knaster-Kuratowski-Mazurkiewicz theorem to variational inequalities, J. Math. Anal. Appl. 137 (1989), 46-58.

[3] Bardaro, C. and Ceppitelli, R., Fixed point theorems and vector-valued minimax theorems, J. Math. Anal. Appl. 146 (1990), 363-373.

[4] Brézis, H., Nirenberg, L. and Stampacchia, G., A remark on Ky Fan's minimax principle, Bollettino U.M.I. 6:4 (1972), 293-300

[5] Chowdhury, M.S.R. and Tan, K.K., Generalization of Ky Fan's minimax inequality with applications to generalized variational inequalities for pseudomonotone operators and fixed point theorems, J. Math. Anal. Appl. 204 (1996), 910-929.

[6] Ding, X.P. and Tan, K.K., A minimax inequality with applications to existence of equilibrium point and fixed point theorems, Colloquium Mathematicum 63 (1992), 233-247.

[7] Fan, K., A minimax inequality and applications, Inequalities III (1972), (ed. by O. Shisha), Academic Press, 103-113.

[8] Horvath, C.D., Points fixes et coincidences pour les applications multivoques sans convexité, C.R. Acad. Sci. Paris 296 (1983), 403-406.

[9] Horvath, C.D., Contractibility and generalized convexity, J. Math. Anal. Appl. 156 (1991), 341-357.

[10] Knaster, B., Kuratowski, C. and Mazurkiewicz, S., Ein beweis des fixpunktsatzes für n-dimensionale simplexe, Fund. Math. 14 (1929), 132-137.

[11] Park, S., Theory of multifunctions on generalized convex spaces, preprint (1993).

[12] Park, S. and Kim, H., Admissible classes of multifunctions on generalized convex spaces, Proc. Coll. Natur. Sci. Seoul Nat. Univ. 18 (1993), 1-21.

[13] Shih, M.H. and Tan, K.K., A further generalization of Ky Fan's minimax inequality and its applications, Studia Mathematica 78 (1984), 279-287.

[14] Tan, K.K., Lecture notes on topics in Topology and Functional Analysis, unpub- 
lished 1985, 1991 and 1994.

[15] Tan, K.K. and Yuan, X.Z., A minimax inequality with applications to existence of equilibrium points, Bull. Austral. Math. Soc. 47 (1993), 483-503.

[16] Tan, K.K. and Zhang, X.L., Fixed point theorems on $G$-convex spaces and applications, Proc. of the First Intern. Conf. on Nonlinear Functional Analysis and Appl., Kyungnam Univ., Masan, Korea 1 (1996), 1-19. 


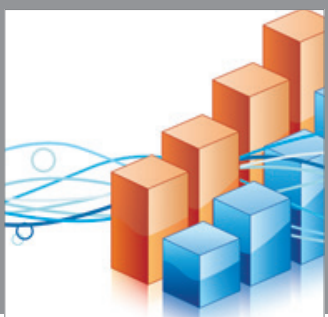

Advances in

Operations Research

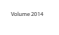

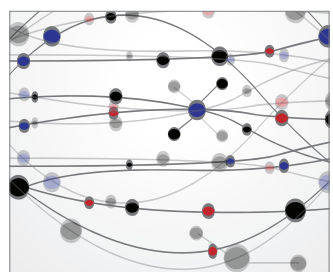

\section{The Scientific} World Journal
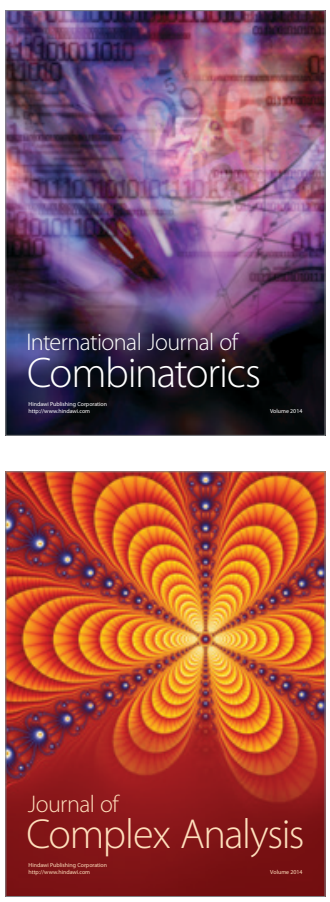

International Journal of

Mathematics and

Mathematical

Sciences
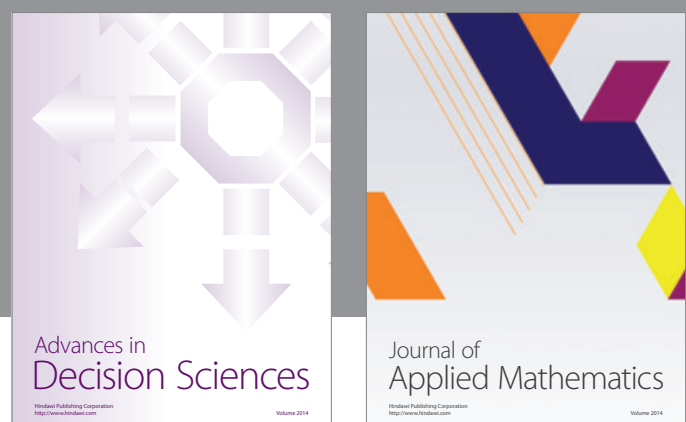

Journal of

Applied Mathematics
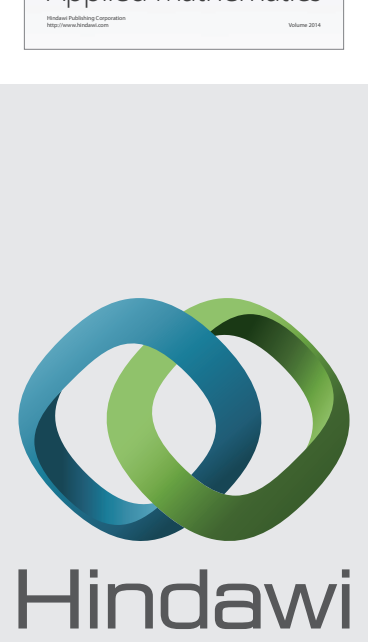

Submit your manuscripts at http://www.hindawi.com
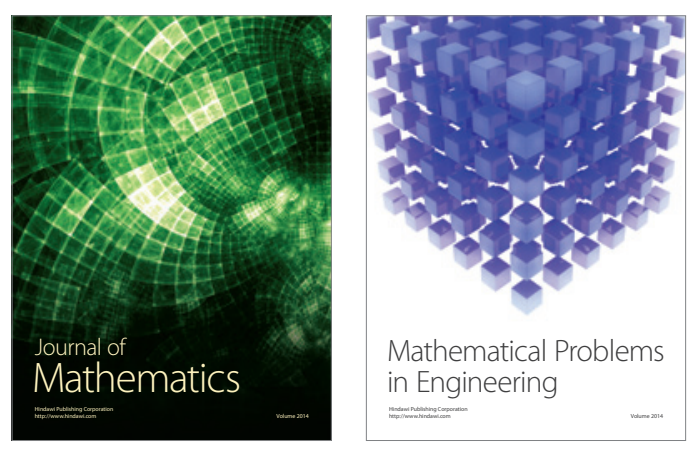

Mathematical Problems in Engineering
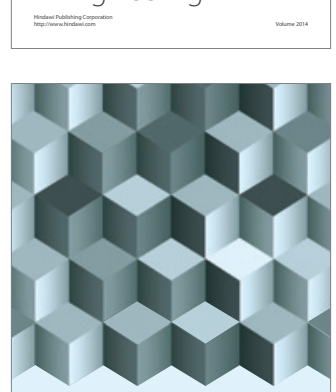

Journal of

Function Spaces
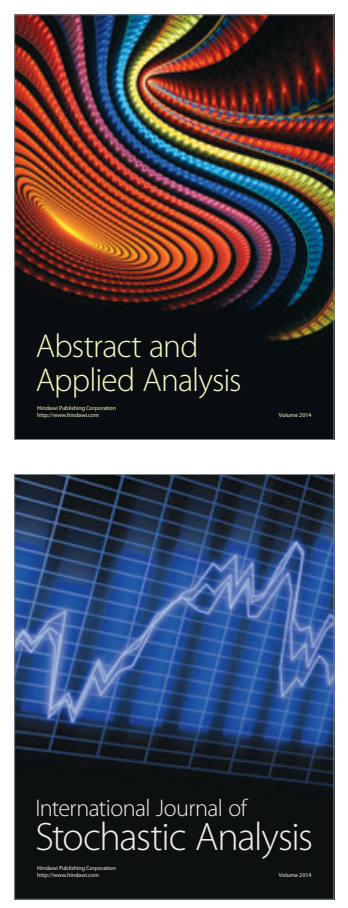

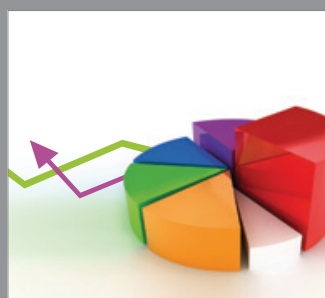

ournal of

Probability and Statistics

Promensencen
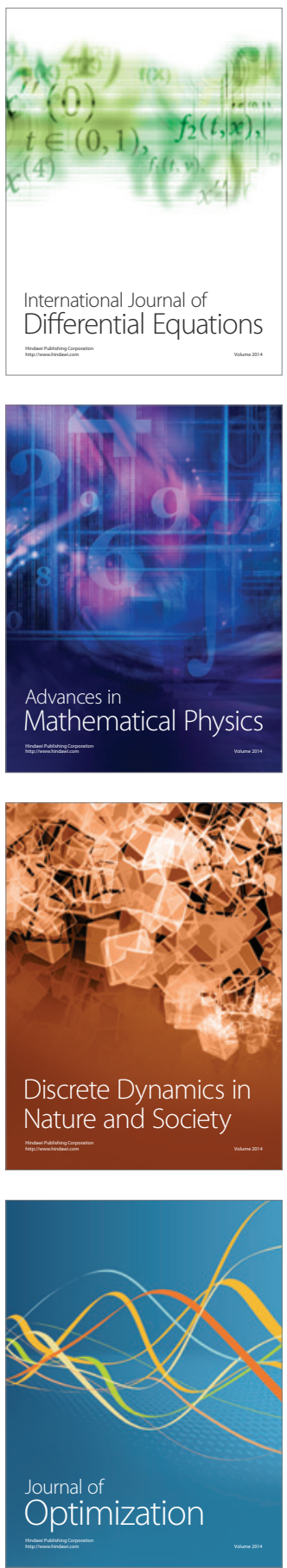